Pacific Journal of Mathematic 


\title{
SUPERCUSPIDAL COMPONENTS OF THE QUATERNION WEIL REPRESENTATION OF $\mathrm{SL}_{2}(\mathrm{f})$
}

\author{
C. Asmuth AND J. REPKA
}

\begin{abstract}
Let $f$ be a $p$-adic field of odd residual characteristic. It is known that all but one summand of the quaternion Weil representation are supercuspidal. These summands are precisely identified in terms of corresponding summands of quadratic extension Weil representations.
\end{abstract}

1. Let $\mathfrak{f}$ be a $p$-adic field of odd residual characteristic. From [6] we know that all supercuspidal representations of $G=\mathrm{SL}_{2}(\mathfrak{f})$ occur as summands of various Weil representations associated with quadratic extensions of $\mathfrak{k}$. It is also known that the Weil representation associated to the unique quaternion division algebra over $\mathfrak{f}$ decomposes into a direct sum of irreducible representations, all but one of which are supercuspidal. The object of this paper is to show just how these representations correspond to summands of quadratic extension Weil representations. The methods depend heavily on [3]. The primary motivation for this paper was the problem of decomposing tensor products of certain supercuspidal representations of $G$. The authors have been told that some similar computations have been worked out by J. Shalika and W. Casselman.

2. In this paper, the ring of integers in $\mathfrak{f}$ and its prime ideal are denoted respectively by $\mathfrak{D}$ and $\mathfrak{p}$. We choose a generator $\pi$ of $\mathfrak{p}$ and a non square unit $\varepsilon$ in $\mathfrak{D}$. The order of the residue class field will be denoted by $q$.

For $\theta \in\{\pi, \varepsilon, \varepsilon \pi\}$, we let $\mathfrak{o}_{0}$ and $\mathfrak{p}_{\theta}$ denote the ring of integers in $\mathfrak{f}(\sqrt{\theta})$ and its prime ideal respectively. Trace and norm of $\mathfrak{f}(\sqrt{\theta})$ over $\mathfrak{t}$ are written $\tau_{\theta}$ and $\nu_{\theta}$ respectively.

The quaternion division algebra over $\mathfrak{f}$ will be denoted by $D$. Its integers will be denoted by $A$ and the prime ideal of $A$ will be $P$. The reduced norm and trace of $D$ over $\mathfrak{t}$ are written respectively $\nu_{D}$ and $\tau_{D}$. The set $\{1, i, j, k\}$ is a basis of $D$ over $\mathfrak{k}$ where $i^{2}=\varepsilon, j^{2}=\pi$, and $i j=-j i=k$. There are convenient imbeddings of $\mathfrak{f}(\sqrt{\varepsilon})$ and $\mathfrak{f}(\sqrt{\pi})$ in $D$ where $\mathfrak{f}(\sqrt{\varepsilon})=\{a+b i: a, b \in \mathfrak{f}\}$ and $\mathfrak{f}(\sqrt{\pi})=\{a+b j: a, b \in \mathfrak{f}\}$. Let $S$ be a complete set of residues of $\mathfrak{v}_{\varepsilon} / \mathfrak{p}_{\varepsilon}$ (and thus of $A / P$ ) consisting of zero and roots of unity. Then for any $z \in D$ we may write $z=\sum_{n=N}^{\infty} \alpha_{n} j^{n}$ where each $\alpha_{n} \in S$.

Since $\pi$ can be chosen to be any element in $\mathfrak{f}$ that generates $\mathfrak{p}$, we will generally consider only the cases $\theta=\varepsilon$ and $\theta=\pi$.

3. From [3], we recall some information on the representations 
of $\Gamma=\left\{\gamma \in D: \nu_{D}(\gamma)=1\right\}$. Let $C^{\theta}=\left\{z \in \mathfrak{f}(\sqrt{\theta}): \nu_{\theta}(z)=1\right\}$. Given $U$ in $\hat{\Gamma}$, there is a natural way of choosing a character $\psi \in \hat{C}^{\theta}$ for some $\theta$. We then say that $U$ is of type $\theta$ and that $U$ corresponds to $\psi$. Let $U$ and, consequently $\theta$ and $\psi$, be fixed. Let $m$ be the smallest integer such that $U$ is trivial on $\Gamma_{m}=\Gamma \cap\left(1+P^{m}\right)$. If $m$ is even, $U$ is of type $\varepsilon \pi$ or $\pi$. If $m$ is odd, $U$ is of type $\varepsilon$. It happens that under this correspondence, no $U \in \hat{\Gamma}$ matches a square trivial character of $C^{\pi}$ or $C^{\epsilon \pi}$.

We will describe $U$ as an induced representation from a subgroup $B(U)$. The inducing representation will have a character $\chi_{U}$ whose degree is either 1 or $q . B(U)$ will always be of the form $C^{\theta} H$ where $H$ is a subgroup of $\Gamma$ depending on $m$. Let $M$ be the smallest odd integer not less than $m$. We shall describe $H$ by giving its elements modulo $\Gamma_{M}$.

First let $\theta=\pi$. Then $m$ is even. If $U$ corresponds to $\psi \in \widehat{C}^{\pi}$, then the conductor of $\psi$ is $C_{s}^{\pi}=C^{\pi} \cap\left(1+\mathfrak{p}_{\pi}^{s}\right)$ where $s=m$. Then $H$ is given by the set of elements $\nu_{D}(\gamma)^{-1 / 2} \gamma$ where $\gamma=1+$ $\sum_{n=s / 2}^{s+1} \alpha_{n} j^{n}$. Each $\alpha_{n} \in S$ and also $\alpha_{n} \in S \cap \mathfrak{t} i$ if $n$ is odd. Thus we may write (modulo $\Gamma_{M}$ )

$$
H=\left\{1+b i j^{s / 2}+i \sum_{n=s / 2+1}^{s+1} b_{n} j^{n}-(-1)^{s / 2} \frac{\varepsilon}{2} b^{2} \pi^{s / 2}: b, b_{n} \in S \cap \mathfrak{l}\right\} .
$$

(When $-1 \notin\left(\mathfrak{k}^{\times}\right)^{2}$, the choice of $U$ may force $j$ and $k$ to be interchanged; this has no effect on the results.) Let $\delta=\alpha h \in B(U)$ where $\alpha \in C^{\pi}$ and $h \in H$. Then $\chi_{U}(\delta)=\psi(\alpha)$ and $\chi_{U}$ is a character of degree one.

Now let $\theta=\varepsilon$. Then $m$ is odd. If $U$ corresponds to $\psi \in \widehat{C}^{\varepsilon}$, then the conductor of $\psi$ is $C_{s}^{\varepsilon}=C^{\varepsilon} \cap\left(1+\mathfrak{p}_{\varepsilon}^{s}\right)$ where $2 s-1=m=M$. Let $H$ be given by those elements $\nu_{D}(\gamma)^{-1 / 2} \gamma$ where $\gamma=1+\sum_{n=s-1}^{2 s-3} \alpha_{n} j^{n}$ and $\alpha_{n} \in S$ is zero when $n$ is even.

Assume first that $s$ is odd. Then $\nu_{D}(\gamma)^{-1 / 2} \equiv 1$ (modulo $\Gamma_{M}$ ) so that $\gamma$ in the form above is in $H$. Here $\chi_{U}$ is of degree one and for $\alpha \in C^{\varepsilon}$, we have $\chi_{U}(\alpha h)=\psi(\alpha)$.

Now let $s$ be even. Then $h \in H$ is in the form $\mu(\beta) h_{0}$ where $\mu(\beta)=1+\beta j^{s-1}+(1 / 2) \nu_{\varepsilon}(\beta) \pi^{s-1}, \quad \beta \in S$, and $h_{0} \in H \cap\left(1+P^{s}\right)$. For $b \in S \cap \mathfrak{t}$, we define $\omega_{M}(b) \in C^{\varepsilon} / C^{\varepsilon} \cap \Gamma_{M}$ by $\omega_{M}(b) \equiv 1+\pi^{s-1} b i$, modulo $1+P^{M}$. Let $\alpha \in C^{\varepsilon}$ be written $\alpha=\alpha_{0} \alpha_{1}$ where $\alpha_{0} \in C^{\varepsilon} \cap S$ and $\alpha_{1} \in$ $C_{1}^{\varepsilon}$. Then we have

$$
\chi_{U}\left(\alpha_{0} \alpha_{1} \mu(\beta) h_{0}\right)=\left\{\begin{array}{l}
-\psi\left(\alpha_{0} \alpha_{1}\right) \psi\left[\omega_{M}\left[\frac{r}{2 t \varepsilon} \nu_{\varepsilon}(\beta)\right]\right], \text { if } \alpha_{0} \neq \pm 1 \\
q \psi\left(\alpha_{0} \alpha_{1}\right), \text { if } \beta=0 \text { and } \alpha_{0}= \pm 1 \\
0, \text { if } \alpha_{0}= \pm 1 \text { and } \beta \neq 0
\end{array}\right.
$$


Here $\alpha_{0}=r+t i . \quad \chi_{U}$ is a character of degree $q$.

4. In this section we give definitions for the Weil representations using formulae from [5]. We fix once and for all a character $\Phi$ of $\mathfrak{l}^{+}$with conductor $\mathfrak{D}$. For $\lambda \in \mathfrak{f}$, let $\Phi_{\lambda}(x)=\Phi(\lambda x)$. Haar measures on the additive groups of $\mathfrak{f}(\sqrt{\theta})$ and $D$ are normalized so that in each case the ring of integers has unit measure.

We let

$$
p(\theta)=\left\{\begin{array}{l}
1, \text { for } \theta=\varepsilon \\
\zeta, \text { for } \theta=\pi
\end{array}\right.
$$

where $\zeta=\sum_{x \varepsilon_{0} / \mathfrak{p}} \Phi\left(\pi^{-1} x^{2}\right)$.

The quadratic extension Weil representations of $G$ will be denoted $T(\theta, \lambda)$. They act on $L^{2}(\mathfrak{f}(\sqrt{\theta}))$. We will define $T(\theta, \lambda)$ on generators of $G$. Let $f \in L^{2}(\mathfrak{t}(\sqrt{\theta}))$. Then

$$
\begin{gathered}
{\left[T(\theta, \lambda)\left(\begin{array}{ll}
1 & b \\
0 & 1
\end{array}\right) f\right](z)=\Phi_{\lambda}\left(b \nu_{\theta}(z)\right) f(z)} \\
{\left[T(\theta, \lambda)\left(\begin{array}{rr}
0 & 1 \\
-1 & 0
\end{array}\right) f\right](z)=c(\theta, \lambda) p(\theta) \operatorname{sgn}_{\theta}(-\lambda) \int_{\mathfrak{t}(\sqrt{\bar{v})}} f(w) \Phi_{\lambda}\left(\tau_{\theta} \bar{z} w\right) d w}
\end{gathered}
$$

where $c(\theta, \lambda)>0$ is a constant chosen so as to make the second operator unitary.

The quaternion Weil representation is denoted $T(D, \lambda)$ (although all choices of $\lambda$ give equivalent representations). Let $f \in L^{2}(D)$. Then

$$
\begin{gathered}
{\left[T(D, \lambda)\left(\begin{array}{ll}
1 & b \\
0 & 1
\end{array}\right) f\right](z)=\Phi_{\lambda}\left(b \nu_{D}(z)\right) f(z)} \\
{\left[T(D, \lambda)\left(\begin{array}{rr}
0 & 1 \\
-1 & 0
\end{array}\right) f\right](z)=-c(D, \lambda) \int_{D} f(w) \Phi_{\lambda}\left(\tau_{D}(\bar{z} w)\right) d w .}
\end{gathered}
$$

Again $c(D, \lambda)>0$ is chosen so that the second operator is unitary. We have the well known decomposition (see [2] or [7])

$$
T(\theta, \lambda)=\frac{1}{\psi \in \hat{C}^{\theta}} T(\theta, \lambda, \psi)
$$

where the representation space of $H(\theta, \lambda, \psi)$ of $T(\theta, \lambda, \psi)$ is given by

$$
\left\{f \in L^{2}(\mathfrak{f}(\sqrt{\theta})): \forall z \in \mathfrak{H}(\sqrt{\theta}), \quad \forall \alpha \in C^{\theta}, f(z \alpha)=f(z) \psi(\alpha)\right\} .
$$

Similarly 


$$
T(D, \lambda)=\frac{1}{U \in \hat{\Gamma}} T(D, \lambda, U) .
$$

Let $\theta_{U}$ be the character of $U$. Then the representation space of $T(D, \lambda, U)$ is

$$
H(U)=\left\{f \in L^{2}(D): \int_{\Gamma} f(z \gamma) \overline{\theta_{U}(\gamma)} d \gamma=f(z)\right\} .
$$

For $U \neq 1, H(U)$ consists of supercuspidal summands. We can write the vector space sum

$$
H(U)=H^{1}(U) \oplus H^{\varepsilon}(U) \oplus H^{\pi}(U) \oplus H^{\varepsilon \pi}(U) .
$$

Here $H^{i}(U)=\left\{f \in H(U): f(x) \neq 0 \Rightarrow \nu_{D}(x) \in \delta\left(\mathfrak{t}^{*}\right)^{2}\right\}$. Now suppose $U$ is of type $\theta$ for some $\theta \in\{\varepsilon, \pi, \varepsilon \pi\}$. Let $\left\{\theta^{\prime}, \theta^{\prime \prime}\right\}=\{\varepsilon, \pi, \varepsilon \pi\}-\{\theta\}$. From Proposition 1.5 of [4], we may conclude that $H^{1}(U) \oplus H^{\theta}(U)$ and $H^{\theta^{\prime}}(U) \oplus H^{\theta^{\prime \prime}}(U)$ are both $G$-invariant. (See also Lemma 4.1 of [3].)

5. From [5] and [7], we know that all supercuspidal representations of $G$ are induced from some compact open subgroup. For suitable $\lambda$, we may assume that $T(\theta, \lambda, \psi)$ is induced from $K=\operatorname{SL}_{2}(\mathrm{p})$. Let the inducing representation be denoted by $S(\theta, \lambda, \psi)$. The object of this section is to give explicit formulae for matrix coefficients of $S(\theta, \lambda, \psi)$ at generators of $K$. To do this we must pick out an orthonormal basis of the representation space of $S(\theta, \lambda, \psi)$.

Let $\psi$ have conductor $C_{s}^{\theta}=C^{\theta} \cap\left(1+\mathfrak{p}_{\theta}^{s}\right)$. We will exclude the cases where $\psi \in \widehat{C}^{\pi}$ (or $\hat{C}^{\varepsilon \pi}$ ) and $\psi^{2} \equiv 1$. Then choose $\lambda$ to be of order $n$ (i.e., $\lambda=u \pi^{n}$ where $u \in \mathfrak{o}^{\times}$) where

$$
n= \begin{cases}-s, & \text { if } \theta=\varepsilon \\ -\left(\frac{s}{2}+1\right), & \text { if } \theta=\pi .\end{cases}
$$

From [2] or [7] we see that this is the "suitable choice" of $\lambda$. Now set

$$
n^{\prime}= \begin{cases}s, & \text { if } \theta=\varepsilon \\ s+1, & \text { if } \theta=\pi .\end{cases}
$$

Let $H_{\theta}(s)$ be the space of all functions supported on $\mathrm{o}_{\theta}$ and constant on cosets of $\mathfrak{p}_{\theta}^{n^{\prime}}$. Let $H_{0}(\theta, \lambda, \psi)=H_{\theta}(s) \cap H(\theta, \lambda, \psi)$. $H_{0}(\theta, \lambda, \psi)$ is then the representation space of $S(\theta, \lambda, \psi)$. The action is simply the action of $T(\theta, \lambda, \psi)$ restricted to $K$.

We now construct an orthonormal basis of $H_{0}(\theta, \lambda, \psi)$. Let $J_{\theta}(s)$ be a complete set of orbit representatives in $\mathrm{o}_{\theta} / \mathfrak{p}_{\theta}^{n^{\prime}}-\pi \mathrm{D}_{\theta} / \mathfrak{p}_{\theta}^{n^{\prime}}$ 
under multiplication by elements of $C^{\theta}$. For each $z \in J_{\theta}(s)$, we define

$$
f_{z}(x)= \begin{cases}r(s, \theta) \xi(\alpha), & \text { for } x \in z \alpha\left(1+\mathfrak{p}_{\theta}^{n^{\prime}}\right) \\ 0, & \text { elsewhere }\end{cases}
$$

Here, $r(s, \theta)>0$ is chosen to make $f_{z}$ a unit vector. We do not need to compute it explicitly.

For $x$ and $y \in J_{0}(s)$, let $m_{x y}^{\psi}(g)=\left\langle S(\theta, \lambda, \dot{\psi})(g) f_{x} \mid f_{y}\right\rangle$. We will now compute $m_{x y}^{2}$ for generators of $K=\mathrm{SL}_{2}(\mathfrak{D})$. First let $g=\left[\begin{array}{ll}1 & b \\ 0 & 1\end{array}\right]$ where $b \in \mathfrak{D}$. Then

$$
m_{x y}^{\psi}(g)= \begin{cases}\Phi_{\lambda}\left(b \nu_{\theta}(x)\right), & \text { if } x=y \\ 0, & \text { if } x \neq y\end{cases}
$$

Now let $g=\left(\begin{array}{rr}0 & 1 \\ -1 & 0\end{array}\right)$. We write

$$
m_{x y}^{\psi}(g)=\int_{t\left(s^{\prime} \theta\right)}\left[\left(\begin{array}{rr}
0 & 1 \\
-1 & 0
\end{array}\right) f_{x}\right](z) \overline{f_{y}(z)} d z
$$

Let $v_{\theta}\left(n^{\prime}\right)$ be the measure of $\mathfrak{p}_{\theta}^{n^{\prime}}$. Then

$$
m_{x y}^{i r}(g)=v_{\theta}\left(n^{\prime}\right) c(\theta, \lambda) p(\theta) \operatorname{sgn}_{\theta}(-\lambda) \sum_{\alpha \in C^{\theta} \mid C_{s}^{\prime}} \psi(\alpha) \Phi_{\lambda}\left(\tau_{\theta}(\bar{y} x \alpha)\right)
$$

Since

$$
c(\theta, \lambda)= \begin{cases}q^{s / 2}, & \text { if } \theta=\pi \\ q^{s}, & \text { if } \theta=\varepsilon\end{cases}
$$

we have

Proposition 5.1. Let

$$
\dot{\phi}(s)= \begin{cases}-\frac{s}{2}-1, & \text { if } \theta=\pi \\ -s, & \text { if } \theta=\varepsilon .\end{cases}
$$

Then

$$
m_{x y}^{\psi}\left(\begin{array}{rr}
0 & 1 \\
-1 & 0
\end{array}\right)=q^{\phi(s)} p(\theta) \operatorname{sgn}_{\theta}(-\lambda) \sum_{\alpha \in C^{\theta} / C_{s}^{\theta}} \dot{\psi}(\alpha) \Phi_{\lambda}\left(\tau_{\theta}(\bar{y} x \alpha)\right) .
$$

6. To describe the representations $T(D, \lambda)$, we wish to find their irreducible subrepresentations. Since the representations $S(\theta, \lambda, \psi)$ induce irreducibly from $K$, to find copies of $T(\theta, \lambda, \psi)$ in a representation it suffices to look for copies of $S(\theta, \lambda, \psi)$ in its 
restriction to $K$. This we shall do by comparing matrix coefficient functions.

We shall try, insofar as possible, to imitate for the representation $T(D, \lambda)$ the construction of $\S 5$. We shall define vectors $F_{z}$ which transform nicely under the restriction of $T(D, \lambda)$ to $K$ and then study the corresponding coefficient functions.

Let $U$ correspond to a character $\psi$ of $C^{\theta}$ with conductor $C_{s}^{\theta}$. Choose $\lambda$ of an order determined by $s$ as in $\S 5$. As in $\S 3$, let

$$
M=\left\{\begin{array}{l}
2 s-1, \text { if } \theta=\varepsilon \\
s+1, \text { if } \theta=\pi
\end{array}\right.
$$

so that $\Gamma_{M}$ is the largest congruence subgroup of $\Gamma$ contained in the kernel of $U$. Let the set of $C^{\theta}$-orbit representatives $J_{\theta}(s)$ be imbedded in $D$ in the natural way mentioned in $\S 2$. For $z \in J_{\theta}(s)$, set

$$
F_{z}(x)= \begin{cases}\chi_{U}(\gamma) R(s) q^{-V(z) / 2}, & \text { for } x \in z \gamma\left(1+P^{M}\right) \\ 0, & \text { elsewhere } .\end{cases}
$$

Here $R(s)>0$ is chosen to make $\left\|F_{z}\right\|_{2}=1$ and $P^{V(z)}$ is the smallest power of $P$ containing $z$.

It should be noted here that the $F_{z}$ 's do not necessarily span a $K$-invariant subspace of $H^{1}(U) \oplus H^{\theta}(U)$. The unfortunate case is when $\theta=\varepsilon$ and $s$ is even. We shall disregard this problem for now and go on to compute matrix coefficients in all cases.

For $g \in K$, let $M_{x y}^{U}(g)=\left\langle T(D, \lambda)(g) F_{x} \mid F_{y}\right\rangle$. When $g=\left(\begin{array}{ll}1 & b \\ 0 & 1\end{array}\right)$ we get an easy result:

Lemma 6.1. Let $U$ correspond to $\psi \in \hat{C}^{\theta}$ and let $\phi \in \hat{C}^{\theta}$ be any character with the same conductor as $\psi$. Then $M_{x y}^{U}\left(\begin{array}{ll}1 & b \\ 0 & 1\end{array}\right)=m_{x y}^{\phi}\left(\begin{array}{ll}1 & b \\ 0 & 1\end{array}\right)$ where $b \in \mathfrak{D}$.

Proof.

$$
M_{x y}^{U}\left(\begin{array}{ll}
1 & b \\
0 & 1
\end{array}\right)= \begin{cases}\Phi_{\lambda}\left(b \nu_{D}(x)\right), & \text { if } x=y \\
0, & \text { if } x \neq y\end{cases}
$$

Since for $x \in J_{\theta}(s), \nu_{D}(x)=\nu_{\theta}(x)$, the result follows.

Thus to distinguish the various representations, we must evaluate the coefficient functions at $g=\left(\begin{array}{rr}0 & 1 \\ -1 & 0\end{array}\right)$. Roughly speaking, this element acts as a Fourier transform, so we are obliged to compute various character sums. Recall the groups $H$ and $B(U)$; $H$ was defined in $\S 3$ and then $B(U)$ was defined by $B(U)=C^{\theta} H$. Let $\mathscr{B}_{M}(U)=B(U) /\left(1+P^{M}\right)$. Then 


$$
\begin{aligned}
M_{x y}^{U}(g) & =\int_{D}\left[\left(\begin{array}{rr}
0 & 1 \\
-1 & 0
\end{array}\right) F_{x}\right](w) \overline{F_{y}(w)} d w \\
& \left.=R(s) q^{-((1 / 2) V(y)+2 M)} \sum_{\gamma \in \mathscr{F}_{M}(U)}\left[\left(\begin{array}{rr}
0 & 1 \\
-1 & 0
\end{array}\right)\right] F_{x}\right](y \gamma) \overline{\chi_{U}(\gamma)} .
\end{aligned}
$$

Now $c(D, \lambda)=q^{M}$ so

$$
\begin{aligned}
& M_{x y}^{U}(g)=-R(s)^{2} q^{-((1 / 2) V(x y)+3 M)} \sum_{\gamma, \hat{o} \in \mathscr{O}_{M}(U)} \chi_{U}(\delta) \Phi_{\lambda}\left(\tau_{D} \bar{y} x \delta \bar{\gamma}\right) \overline{\chi_{U}(\gamma)} \\
& =-R(s)^{2} q^{-((1 / 2) V(x y)+3 M)}\left|\mathscr{B}_{M}(U)\right|\left(\operatorname{deg} \chi_{U}\right)^{-1} \sum_{\delta \in \mathscr{B}_{M}(U)} \chi_{U}(\delta) \Phi_{\lambda}\left(\tau_{D} \bar{y} x \delta\right) .
\end{aligned}
$$

Since $R(s)^{2} q^{-2 M}\left|\mathscr{B}_{M}(U)\right|=1$ we have

LEMMA 6.2.

$$
M_{x y}^{U}\left(\begin{array}{rr}
0 & 1 \\
-1 & 0
\end{array}\right)=-q^{-((1 / 2) V(x y)+M)}\left(\operatorname{deg} \chi_{U}\right)^{-1} \sum_{\delta \in \mathscr{B}, M} \chi_{U}(\gamma) \Phi_{\lambda}\left(\tau_{D} \bar{y} x \delta\right)
$$

It is this formula which we must evaluate more fully. We now consider several cases. First let $\theta=\pi$ and assume that $x$ and $y$ are units in $J_{\pi}(s)$. Then

$$
M_{x y}^{U}\left(\begin{array}{rr}
0 & 1 \\
-1 & 0
\end{array}\right)=-q^{-(s+1)} \sum_{\delta \in \mathscr{G}_{M}(U)} \chi_{U}(\gamma) \Phi_{\lambda}\left(\tau_{D} \bar{y} x \delta\right)
$$

From $\S 3$ we see that the elements of $\mathscr{B}(U)$ can be identified with pairs $(\alpha, h)$ where $\alpha \in C^{\pi} / C_{s}^{\pi}$ and $h \in \mathscr{H}=H /\left(1+P^{M}\right)$. Morever elements of $\mathscr{H}$ can be expressed in the form

$$
1+b i j^{s / 2}-(-1)^{s / 2} \frac{\varepsilon}{2} b^{2} \pi^{s / 2}+i \sum_{n=s / 2+1} b_{n} j^{n}
$$

where for all $n, b_{n} \in S \cap \mathfrak{t}$. Thus

$$
M_{x y}^{U}\left(\begin{array}{rr}
0 & 1 \\
-1 & 0
\end{array}\right)=-q^{-(s+1)} \sum_{\alpha \in C^{\pi} / C_{s}^{\pi}} \psi(\gamma) \Phi_{\lambda}\left(\tau_{\pi} \bar{y} x \alpha\right) \sum_{h \in \mathscr{C}} \Phi_{\lambda}\left(\tau_{D} \bar{y} x \alpha(h-1)\right)
$$

where $U$ corresponds to $\psi \in \widehat{C}^{\pi}$. Consider the inside sum over $\mathscr{H}$.

$$
(*)=\sum_{h \in \mathscr{C}} \Phi_{\lambda}\left(\tau_{D} \bar{y} x \alpha(h-1)\right)=q^{s / 2} \sum_{b \in S \cap t} \Phi_{\lambda}\left(-y x \widetilde{\alpha}(-1)^{s / 2} \varepsilon \pi^{s / 2} b^{2}\right)
$$

where $\tilde{\alpha}= \pm 1$ is the image of $\alpha$ modulo $C_{1}^{\pi}$. Now let $\sigma$ be the character of order 2 on the group of units of $\mathfrak{D}_{\pi}$ whose kernel is the squares. Then

$$
(*)=-\sigma(\bar{y} x) \sigma(\alpha) \operatorname{sgn}_{\pi}(-\lambda) p(\pi) q^{s / 2} .
$$


Thus we have

Lemma 6.3. Let $\theta=\pi$ and assume that $x, y \in \mathfrak{p}_{\pi}^{\times} \cap J_{\pi}(s)$. Then with $\sigma$ as above and $U \in \hat{\Gamma}$ corresponding to $\psi \in \widehat{C}^{\pi}$ we have

$$
M_{x y}^{U}\left(\begin{array}{rr}
0 & 1 \\
-1 & 0
\end{array}\right)=\sigma(\bar{y} x) q^{-(s / 2)-1} p(\pi) \operatorname{sgn}_{\pi}(-\lambda) \sum_{a \in C^{\pi} / C_{s}^{\pi}}(\psi \sigma)(\alpha) \Phi_{\lambda}\left(\tau_{\pi}(\bar{y} x \alpha)\right) .
$$

LEMmA 6.4. If $x$ and $y$ are both elements of $\mathfrak{p}_{\pi}$, then

$$
m_{x y}^{\psi \sigma}\left(\begin{array}{rr}
0 & 1 \\
-1 & 0
\end{array}\right)=M_{x y}^{U}\left(\begin{array}{rr}
0 & 1 \\
-1 & 0
\end{array}\right)=0
$$

where $U$ corresponds to $\psi$ as before.

Proof. If either $x$ or $y$ is in $\mathfrak{p}_{\pi}$, the expression (*) no longer depends on $\alpha$. Thus

$$
\sum_{\alpha \in C \pi / C_{s}^{\pi}}(\psi \sigma)(\alpha) \Phi_{2}\left(\tau_{\pi}(\bar{y} x \alpha)\right)
$$

is a common factor of $m_{x y}^{\psi r \sigma}$ and $M_{x y}^{U}$. If both $x$ and $y$ are in $\mathfrak{p}_{\pi}$, then this factor is zero since $\Phi_{\lambda}\left(\tau_{\pi}(\bar{y} x \alpha)\right)$ is constant on cosets of a subgroup of $C^{\pi}$ on which $\psi \sigma$ is nontrivial.

Now let $\theta=\varepsilon$. Thus we take $U \in \hat{\Gamma}$ to be of type $\varepsilon$ corresponding to $\psi \in \widehat{C}^{\varepsilon}$. Let $\psi$ have conductor $C^{\varepsilon} \cap\left(1+\mathfrak{p}_{\varepsilon}^{s}\right)$. Then set $M=$ $2 s-1$. We consider cases according to the parity of $s$. First let $s$ be odd. Then $\operatorname{deg} \chi_{U}=1$. A simple computation gives

$$
\begin{aligned}
M_{x y}^{U}\left(\begin{array}{rr}
0 & 1 \\
-1 & 0
\end{array}\right) & =-q^{-(2 s-1)} \sum_{\delta \in \mathscr{F}_{M}(U)} \chi_{U}(\delta) \Phi_{\lambda}\left(\tau_{D} \bar{y} x \delta\right) \\
& =-q^{-(2 s-1)} \sum_{\alpha \in C^{\varepsilon} / C^{\varepsilon}}\left[\psi(\alpha) \Phi_{\lambda}\left(\tau_{\varepsilon} \bar{y} x \alpha\right) \sum_{\mathscr{O}} \Phi_{\lambda}\left(\tau_{D} \bar{y} x \alpha(h-1)\right)\right] .
\end{aligned}
$$

Now $\lambda$ is chosen according to the prescription in $\S 5$. Thus for $s$ odd, $\operatorname{sgn}_{\varepsilon}(-\lambda)=-1$. In this case, $\Phi_{\lambda}\left(\tau_{D} \bar{y} x \alpha(h-1)\right)=1$ for all $h \epsilon$ $\mathscr{H}$. Thus we have

Lemma 6.5. Let $s$ be odd. Then

$$
M_{x y}^{U}\left(\begin{array}{rr}
0 & 1 \\
-1 & 0
\end{array}\right)=\operatorname{sgn}_{\varepsilon}(-\lambda) p(\varepsilon) q^{-s} \sum_{\alpha} \psi(\alpha) \Phi_{\lambda}\left(\tau_{\varepsilon} \bar{y} x \alpha\right) .
$$

The case when $s$ is even is more involved. From $\S 3$, we recall that $\operatorname{deg} \chi_{U}=q$. Therefore by Lemma 6.2 


$$
M_{x y}^{U}\left(\begin{array}{rr}
0 & 1 \\
-1 & 0
\end{array}\right)=-q^{-2 s} \sum_{\delta \in \mathscr{O}_{M}(U)} \chi_{U}(\delta) \Phi_{\lambda}\left(\tau_{D} \bar{y} x \delta\right)
$$

We shall simplify this sum and find

Lemma 6.6. Let $s$ be even. Then

$$
M_{x y}^{U}\left(\begin{array}{rr}
0 & 1 \\
-1 & 0
\end{array}\right)= \begin{cases}\operatorname{sgn}_{\varepsilon}(-\lambda) p(\varepsilon) q^{-s} \sum_{\alpha \in C^{\varepsilon} / C_{s}^{\varepsilon}} \psi(\alpha) \Phi_{\lambda}\left(\tau_{\varepsilon} \bar{y} x \alpha\right), & \text { if } \bar{y} x \in \mathfrak{t} \\
-q^{-s-1} \sum_{\alpha \in C^{\varepsilon} / C_{s}^{\varepsilon}} \psi(\alpha) \Phi_{\lambda}\left(\tau_{\varepsilon} \bar{y} x \alpha\right), & \text { if } \bar{y} x \notin \mathfrak{t} .\end{cases}
$$

Proof. We need to find an acceptable parametrization of the elements of $\mathscr{B}_{M}(U)$. Recall that $\delta \in B(U)$ is of the form $\delta=\alpha h$ where $\alpha \in C^{\varepsilon}$ and $h \in H$. For $\alpha \in C^{\varepsilon}$, we have $\alpha=\alpha_{0} \rho \omega_{M}(b)$ (modulo $\left.C_{s}^{\varepsilon}\right)$ where $\alpha_{0} \in S \cap C^{\varepsilon}, \rho$ is a representative of $C_{s-1}^{\varepsilon}$ in $C_{1}^{\varepsilon}$, and $\omega_{M}(b) \in$ $C_{s}^{\varepsilon}$ is as defined in $\S 3$. For $h \in H$ we have $h=\mu(\beta) h_{0}$ where $\mu(\beta)=$ $1+\beta_{j}^{s-1}+(1 / 2) \varepsilon \varepsilon_{\varepsilon}(\beta) \pi^{s-1}, \beta \in S$, and $h_{0} \in H \cap 1+P^{s}$. As before, let $\mathscr{H}=H / \Gamma_{M}$ and also let $\mathscr{C}_{0}=\left[H \cap\left(1+P^{s}\right)\right] / \Gamma_{M}$. $\mathscr{C}_{0}$ is given by elements of the form

$$
1+\sum_{n=s+1}^{2 s-2} \beta_{n} j^{n}
$$

where $\beta_{n} \in S$. Now

$$
M_{x y}^{U}\left(\begin{array}{rr}
0 & 1 \\
-1 & 0
\end{array}\right)=-q^{-2 s} \sum_{\alpha \in \mathcal{C}^{\varepsilon} / I_{s}^{\varepsilon}} \sum_{h \in \mathscr{Q} C} \chi_{U}(\alpha h) \Phi_{\lambda}\left(\tau_{D} \bar{y} x \alpha\right) \Phi_{y}\left(\tau_{D} \bar{y} x \alpha(h-1)\right) .
$$

We choose $v \in \mathfrak{D}^{\times}$such that $\psi\left(\omega_{M}(b)\right)=\Phi_{\lambda}\left(\pi^{s-1} v b\right)$. Let $Z_{s}^{\varepsilon}=C^{\varepsilon} / C_{s}^{\varepsilon}-$ $\left[ \pm C_{1}^{\varepsilon} / C_{s}^{\varepsilon}\right]$. Then

$$
\begin{gathered}
M_{x y}^{U}\left(\begin{array}{rr}
0 & 1 \\
-1 & 0
\end{array}\right)=-q^{-2 s} \sum_{\alpha € \pm C_{1}^{\varepsilon} / C_{s}^{s}} q \psi(\alpha) \Phi_{\lambda}\left(\tau_{D} \bar{y} x \alpha\right) \sum_{h \in \mathscr{P}} \Phi_{\lambda}\left(\tau_{D} \bar{y} x \alpha(h-1)\right) \\
-q^{-2 s} \sum_{\alpha \in Z_{s}^{\varepsilon}} \chi_{U}(\alpha h) \Phi_{\lambda}\left(\tau_{D} \bar{y} x \alpha\right) \Phi_{\lambda}\left(\tau_{D} \bar{y} x \alpha(h-1)\right) .
\end{gathered}
$$

Let us assume for the moment that $\bar{y} x \in \mathfrak{f}$. It is easy to check that for $\alpha \in \pm C_{1}^{\varepsilon}$, the expression $\Phi_{\lambda}\left(\tau_{D} \bar{y} x \alpha\right) \sum_{h \in \mathscr{C}} \Phi_{\lambda}\left(\tau_{D} \bar{y} x \alpha(h-1)\right)$ is constant on cosets of $C_{s-1}^{\varepsilon}$. Hence the first sum in (**) vanishes. Thus

$$
M_{x y}^{U}\left(\begin{array}{rr}
0 & 1 \\
-1 & 0
\end{array}\right)=-q^{-2 s} \sum_{\alpha \in Z_{s}^{\varepsilon}} \sum_{h \in \mathscr{\partial} C} \chi_{U}(\alpha h) \Phi_{\lambda}\left(\tau_{D} \bar{y} x \alpha\right) \Phi_{\lambda}\left(\tau_{D} \bar{y} x \alpha(h-1)\right) .
$$

Let $\alpha \in Z_{s}^{\varepsilon}$ be given in the form $\alpha_{0} \rho \omega_{M}(b)$ where $\alpha_{0} \neq \pm 1, \rho \in C_{1}^{\varepsilon} /$ $C_{s-1}^{\varepsilon}$, and $\omega_{M}(b) \in C_{s-1}^{\varepsilon} / C_{s}^{\varepsilon}$. Let $\alpha_{0}=r+t i$. This yields 


$$
\begin{array}{r}
M_{x y}^{U}\left(\begin{array}{rr}
0 & 1 \\
-1 & 0
\end{array}\right)=\sum_{\alpha_{0} \neq \pm 1}\left(\sum_{\rho, b}-\psi\left(\alpha_{0} \rho\right) \Phi_{\lambda}\left(\pi^{s-1} v b\right) \Phi_{\lambda}\left(\tau_{D} \bar{y} x \alpha_{0} \rho \omega_{M}(b)\right)\right) \\
\cdot\left(\sum_{\beta \in S} \sum_{h \in \mathscr{C}_{0}} \Phi_{\lambda}\left(\pi^{s-1} \frac{v r}{2 t \varepsilon} \nu_{\varepsilon}(\beta)\right) \Phi_{\lambda}\left(\tau_{D} \bar{y} x \alpha_{0} \frac{1}{2} \nu_{\varepsilon}(\beta) \pi^{s-1}\right)\right)
\end{array}
$$

The first factor of each term can be written

$$
\sum_{\rho} \sum_{b}-\psi\left(\alpha_{0} \rho\right) \Phi_{\lambda}\left(\pi^{s-1} v b\right) \Phi_{\lambda}\left(2 \pi^{s-1} \bar{y} x t \varepsilon b\right)
$$

Hence if the term corresponding to $\alpha_{0}=r+t i$ is to be nonzero, we will have $-v=2 \bar{y} x t \varepsilon$. This fixes $t$. The second factor then becomes

$$
\sum_{\beta \in S} \sum_{h \in \mathscr{C}_{0}} \Phi_{\lambda}\left(-\pi^{s-1} r \bar{y} x \nu_{\varepsilon}(\beta)\right) \Phi_{\lambda}\left(\tau_{D} \bar{y} x(r+t i) \frac{1}{2} \nu_{\varepsilon}(\beta) \pi^{s-1}\right)
$$

which simplifies to $q^{s}$. Hence

$$
M_{x y}^{U}\left(\begin{array}{rr}
0 & 1 \\
-1 & 0
\end{array}\right)=-q^{-s} \sum_{\alpha \in( \pm r+t \imath) C_{1}^{\varepsilon} / C_{s}^{\varepsilon}} \psi(\alpha) \Phi_{\lambda}\left(\tau_{\varepsilon} \bar{y} x \alpha\right)
$$

But this is just

$$
\operatorname{sgn}_{\varepsilon}(-\lambda) q^{-s} \sum_{\alpha \in C^{\varepsilon} \mid C_{s}^{\varepsilon}} \psi(\alpha) \Phi_{\lambda}\left(\tau_{D} \bar{y} x \alpha\right)
$$

since for $s$ even, $\operatorname{sgn}_{\varepsilon}(-\lambda)=1$ and all terms vanish for which $\alpha \notin$ $( \pm r+t i) C_{1}^{\varepsilon}$.

Now assume $\bar{y} x \notin \mathfrak{t}$, that is, $\bar{y} x=m+n i$ where $n$ is a unit. Consider the formula (**). It is no longer necessarily true that the first sum vanishes. In any case it can be simplified to

$$
-q^{-s-1} \sum_{\alpha \in \pm C_{1}^{\varepsilon /} / C_{s}^{\varepsilon}} \psi(\alpha) \Phi_{\lambda}\left(\tau_{D} \bar{y} x \alpha\right)
$$

The second sum in $\left(^{* *}\right)$ is

$$
\begin{aligned}
-q^{-2 s} & \sum_{\alpha \in Z_{s}^{\varepsilon}} \sum_{h \in \mathscr{C}} \chi_{U}(\alpha h) \Phi_{\lambda}\left(\tau_{D} \bar{y} x \alpha\right) \Phi_{\lambda}\left(\tau_{D} \bar{y} x \alpha(h-1)\right) \\
= & -q^{-2 s} \sum_{\alpha_{0} \neq 1}\left(\sum_{\rho, b}-\psi\left(\alpha_{0} \rho\right) \Phi_{\lambda}\left(\pi^{s-1} v b\right) \Phi_{\lambda}\left(\tau_{D} \bar{y} x \alpha\right)\right) \\
\cdot & \left(\sum_{\beta \in S} \sum_{h \in \mathscr{C}_{0}} \Phi_{\lambda}\left(\pi^{s-1} \frac{v r}{2 t \varepsilon} \nu_{\varepsilon}(\beta)\right) \Phi_{\lambda}\left(\tau_{D} \bar{y} x \alpha \frac{1}{2} \nu_{\varepsilon}(\beta)\right)\right)
\end{aligned}
$$

Reasoning as before, we see that the first factor in each term is zero unless $2 m t \varepsilon+2 n r \varepsilon=-v$. In that event the second factor becomes 


$$
\sum_{h \in \mu_{0}} \sum_{\beta \in S} \Phi_{\lambda}\left(\pi^{s-1}\left(\frac{-n}{t}\right) \nu_{\varepsilon}(\beta)\right)=-q^{s-1}
$$

7. We now state and prove the main result. Let $U \in \hat{\Gamma}$ be chosen where $U$ is nontrivial and of degree $d$. Let $U$ correspond to $\psi \in \hat{C}^{\theta}$ in the manner of $\S 3$. Choose $\lambda \in \mathfrak{f}^{\times}$and set

$$
\lambda^{\prime}=\left\{\begin{array}{l}
\pi \lambda, \text { for } \theta=\varepsilon \\
\varepsilon \lambda, \text { for } \theta \neq \varepsilon .
\end{array}\right.
$$

Let $\sigma \in \hat{\mathfrak{v}}_{\theta}^{\times}$be the character of order 2 whose kernel is the squares. Set

$$
\widetilde{\psi}=\left\{\begin{array}{l}
\psi, \text { if } \theta=\varepsilon \text { or }-1 \in\left(\mathfrak{f}^{\times}\right)^{2} \\
\psi \sigma, \text { if } \theta \neq \varepsilon \text { and }-1 \notin\left(\mathfrak{f}^{\times}\right)^{2}
\end{array}\right.
$$

THEOREM 7.1. $T(D, \lambda, U) \cong d^{2}\left[T(\theta, \lambda, \widetilde{\psi}) \oplus T\left(\theta, \lambda^{\prime}, \widetilde{\psi}\right)\right]$

Lemma 7.2. To prove the theorem, it suffices to consider the case where $\lambda$ is determined by $\psi$ as in $\S 5$.

Proof. Let $\lambda_{1}$ and $\lambda_{2}$ in $\mathfrak{l}^{\times}$be given. Then there exists an element $g_{0} \in \mathrm{GL}_{2}(\mathfrak{f})$ such that $T\left(\theta, \lambda_{1}\right)\left(g_{0} g g_{0}^{-1}\right)=T\left(\theta, \lambda_{2}\right)(g)$ and $T\left(D, \lambda_{1}\right)\left(g g_{0} g g_{0}^{-1}\right)=T\left(D, \lambda_{2}\right)(g)$. (See Lemma 3.3 of [1].)

Now let $U \in \hat{\Gamma}$ be fixed with corresponding $\psi \in \hat{C}^{\theta}$. Let $M$ be determined as in $\S 3$. Let $V_{M}$ be the space of functions on $D$ which are supported on $A$ and are constant on cosets of $P^{M}$. With $\lambda$ chosen as before, we see that $V_{M}$ is a finite-dimensional $K$-invariant subspace of $L^{2}(D)$. For $\delta \in\{1, \varepsilon, \pi, \varepsilon \pi\}$ set $H_{0}^{\delta}(U)=H^{\delta}(U) \cap$ $V_{M}$. Thus $H_{0}^{1}(U) \oplus H_{0}^{\theta}(U)$ is also $K$-invariant. The following lemma can be derived from basic properties of $T(D, \lambda)$.

Lemma 7.3. Assume that $W \subset H^{1}(U) \oplus H^{\theta}(U)$ is isomorphic to some $H(\theta, \lambda, \rho)$. Then

(a) $H^{1}(U) \oplus H^{\theta}(U) \cong d^{2} H(\theta, \lambda, \rho)$.

(b) $H^{\theta^{\prime}}(U) \oplus H^{\theta^{\prime \prime}}(U) \cong d^{2} H\left(\theta, \lambda^{\prime}, \rho\right)$ where $\left\{\theta^{\prime}, \theta^{\prime \prime}\right\}=\{1, \pi, \varepsilon, \varepsilon \pi\}-$ $\{1, \theta\}$.

(c) $H_{0}^{1}(U) \oplus H_{0}^{\theta}(U) \cong d^{2} H_{0}(\theta, \lambda, \rho)$.

Since we know that for $U \neq 1, T(D, \lambda, U)$ consists entirely of supercuspidal summands, we can reduce the problem to that of finding $\rho$.

The method we use is to compare directly the matrices operat- 
ing on $H_{0}(\theta, \lambda, \rho)$ with those operating on $H_{0}^{1}(U) \oplus H_{0}^{\theta}(U)$.

First let $\theta=\varepsilon$ and let $s$ be odd. Then the vectors $F_{y}$ span a $K$-invariant subspace of $H_{0}^{1}(U) \oplus H_{0}^{\varepsilon}(U)$. This is because the matrix given by $M_{x y}^{U}(g)$ is identical with the unitary matrix $m_{x y}^{\psi}(g)$ for all $g \in K$. This additionally shows that $\psi=\rho$ and proves the theorem in this case.

Now assume $\theta=\pi$ (or $\varepsilon \pi$ ). It is not hard to see that the matrices given by $m_{x y}^{\tilde{r}}\left(\begin{array}{rr}0 & 1 \\ -1 & 0\end{array}\right)$ and $M_{x y}^{U}\left(\begin{array}{rr}0 & 1 \\ -1 & 0\end{array}\right)$ are conjugate by a diagonal unitary matrix. Since the matrices for other generators of $K$ are equal and diagonal, the theorem follows.

When $\theta=\varepsilon$ and $s$ is even, we need to say more. When $\bar{y} x \notin \mathfrak{t}$, $M_{x y}^{U}\left(\begin{array}{rr}0 & 1 \\ -1 & 0\end{array}\right)$ is smaller by a factor of $q$ than the corresponding $m_{r y}^{\tilde{\psi}}\left(\begin{array}{rr}0 & 1 \\ -1 & 0\end{array}\right)$. Since all representations are unitary we must conclude that $\left\{F_{x}: x \in J_{\varepsilon}(s)\right\}$ does not span a $K$-invariant space when $s$ is even.

Let $J_{\varepsilon}(s)=X \cup Y$ where $H_{0}^{1}(U)$ contains $\left\{F_{x}: x \in X\right\}$ and $H_{0}^{\varepsilon}(U)$ contains $\left\{F_{y}: y \in Y\right\}$. We can safely assume that $\bar{y} x \in \mathfrak{f}$ if and only if $x$ and $y$ are both in $X$ or both in $Y$. We need to find a set $\left\{\widetilde{F}_{y}: y \in Y\right\}$ such that $\left\{F_{x}: x \in X\right\} \cup\left\{\widetilde{F}_{y}: y \in Y\right\}$ spans a $K$-invariant space. The following lemmas are derived from the constructions of the functions $F_{x}$ and $f_{x}, x \in J_{\varepsilon}(s)$, and the formulae in [4].

LEMma 7.4. $H_{0}^{\varepsilon}(U)$ is the orthogonal direct sum of eigenspaces $W_{y}$ of $\left\{\left(\begin{array}{ll}1 & b \\ 0 & 1\end{array}\right): b \in \mathfrak{0}\right\}$. The dimension of each $W_{y}$ is $d^{2}$. For $y \in Y$, $F_{y} \in W_{y}$ and $W_{y}$ is spanned by the left and right translates of $F_{y}$ by elements in $\Gamma$.

Lemma 7.5. Fix $y_{0} \in Y$. Then for each $y \in Y$ there exists a $g_{y} \in K$ such that

(a) $\left[T(\varepsilon, \lambda, \psi)\left(g_{y}\right)\right]\left(f_{y_{0}}\right)=f_{y}$.

(b) $\left[T(D, \lambda, U)\left(g_{y}\right)\right]\left(F_{y_{0}}\right)=F_{y}$.

Let $W$ be the irreducible $K$-space generated by $\left\{F_{x}: x \in X\right\}$. Since $s \neq 1$ there exists $x$ such that $m_{x y_{0}}^{\psi_{0}}\left(\begin{array}{rr}0 & 1 \\ -1 & 0\end{array}\right) \neq 0$ and therefore $M_{x y_{0}}^{U}\left(\begin{array}{rr}0 & 1 \\ -1 & 0\end{array}\right) \neq 0$. Hence we may pick $\widetilde{F}_{y_{0}} \in W_{y_{0}}$ such that $\widetilde{F}_{y_{0}} \in W$ and $\left\|\widetilde{F}_{y_{0}}\right\|_{2}=1$. For $y \in Y$, set $\widetilde{F}_{y}=\left[T(D, \lambda, U)\left(g_{y}\right)\right]\left(\widetilde{F}_{y_{0}}\right)$. The set $\left\{F_{x}, \widetilde{F}_{y}: x \in X, y \in Y\right\}$ is thus a basis for a $K$-invariant subspace of $H_{0}^{1}(U) \oplus H_{0}^{\varepsilon}(U)$. Let $\left\{\widetilde{M}_{x y}^{U}\right\}$ be the set of matrix coefficient functions on $K$ with respect to this new basis.

LemMA 7.6. There exists a constant $\mu \neq 0$ such that $\widetilde{F}_{y}=\mu F_{y}+$ $P_{y}$ where $P_{y}$ is orthogonal to $F_{y}$. 
Proof. For each $y \in Y,\left\langle\widetilde{F}_{y} \mid F_{y}\right\rangle=\left\langle\widetilde{F}_{y_{0}} \mid F_{y_{0}}\right\rangle$ since $T(D, \lambda, U)\left(g_{y}\right)$ is a unitary transformation. If $\mu$ were zero, then all $M_{x y}^{c}\left(\begin{array}{rr}0 & 1 \\ -1 & 0\end{array}\right)$ would be zero for $x \in X, y \in Y$. Then $m_{x y}^{y}\left(\begin{array}{rr}0 & 1 \\ -1 & 0\end{array}\right)$ would also be zero in these cases. This contradicts the irreducibility of $T(\varepsilon, \lambda, \psi)$ for $\psi^{2} \neq 1$. (See [2] and [7].)

Lemma 7.7. There exists a $K$-space isomorphism $\alpha: F_{y} \rightarrow \widetilde{F}_{y}$ for each $y \in Y$.

Proof. By Lemmas 7.3(c) and 7.4, we can find such $\alpha$ for $y=y_{0}$. Since $\alpha$ commutes with action by $K$ the lemma follows.

LEMMA 7.8. For $x$ and $y$ both in $X$ or both in $Y$ we have $\tilde{M}_{x y}^{\prime \prime}\left(\begin{array}{rr}0 & 1 \\ -1 & 0\end{array}\right)=M_{x y}^{\iota}\left(\begin{array}{rr}0 & 1 \\ -1 & 0\end{array}\right)$.

Proof. For $x$ and $y \in X$ it is clear. For $x$ and $y$ in $Y$, use Lemma 7.7.

Lemma 7.9. Let $x \in X$ and $y \in Y$. Then

$$
\widetilde{M}_{x y}^{L}\left(\begin{array}{rr}
0 & 1 \\
-1 & 0
\end{array}\right)=\mu^{-1} M_{x y}^{U}\left(\begin{array}{rr}
0 & 1 \\
-1 & 0
\end{array}\right)
$$

$P_{r}$ oof. Let $w$ be the projection of $\left[T(D, \lambda, U)\left(\begin{array}{rr}0 & 1 \\ -1 & 0\end{array}\right)\right]\left(F_{x}\right) \quad$ on $W_{y}$. Then $w=\widetilde{M}_{x y}^{v}\left(\begin{array}{rr}0 & 1 \\ -1 & 0\end{array}\right) \widetilde{F}_{y}=\widetilde{M}_{x y}^{v}\left(\begin{array}{rr}0 & 1 \\ -1 & 0\end{array}\right)\left(\mu F_{y}+P_{y}\right)$. Now $\left\langle w \mid F_{y}\right\rangle=$ $M_{x y}^{v}\left(\begin{array}{rr}0 & 1 \\ -1 & 0\end{array}\right)$ by definition and is equal to $\mu \tilde{M}_{x y}^{r}\left(\begin{array}{rr}0 & 1 \\ -1 & 0\end{array}\right)$ by computation.

The fact that the functions $\tilde{M}_{x y}^{v}\left(\begin{array}{rr}0 & 1 \\ -1 & 0\end{array}\right)$ and $m_{x y}^{\psi}\left(\begin{array}{rr}0 & 1 \\ -1 & 0\end{array}\right)$ form unitary matrices forces $|\mu|=q^{-1}$. It is now clear that these matrices are conjugate by some diagonal unitary matrix. Theorem 7.1 follows.

\section{REFERENCES}

1. C. Asmuth, Weil representations of symplectic p-adic groups, Amer. J. Math., 10 (1979), 885-908.

2. W. Casselman, On the representations of $S L_{2}(k)$ related to binary quadratic forms, Amer. J. Math., 94 (1972), 810-834.

3. L. Corwin, Representations of division algebras over local fields, Advances in Mathematics, 13, No. 3, (1974), 259-267.

4. H. Jacquet and R. Langlands, Automorphic Forms on GL(2), Springer Lecture Notes, No. 114, 1970. 
5. M. Saito, Representations unitaires des groupes symplectiques, J. Math. Soc. of Japan, 24 (1972), 232-251.

6. P. Sally and J. Shalika, Characters of the discrete series of representations of SL(2) over a local field, Proc. National Academy of Sciences, 61 (1968), 1231-1237.

7. J. Shalika, Representations of the Two by Two Unimodular Group Over Local Fields, Institute for Advanced Study, Princeton, 1966.

Received November 7, 1979.

TeXas A \& M University

College Station, TX 77843

AND

UNIVERSity OF TORONTO

Toronto, Canada M5S 1A1 


\section{PACIFIC JOURNAL OF MATHEMATICS}

\section{EDITORS}

DONALD BABBITT (Managing Editor)

University of Galifornia

Los Angeles, California 90024

HUgo RossI

University of Utah

Salt Lake City, UT 84112

C. C. MOORE AND ANDREW OGG

University of California

Berkeley, CA 94720

\section{J. DUGUNDJI}

Department of Mathematics University of Southern California Los Angeles, California 90007

R. Finn and J. Milgram

Stanford University

Stanford, California 94305

\section{ASSOCIATE EDITORS}
R. ARENS
E. F. BECKENBACH
B. H. NeUManN
F. WOLF
K. YosHIDA

\section{SUPPORTING INSTITUTIONS}

UNIVERSITY OF ARIZONA

UNIVERSITY OF BRITISH COLUMBIA

CALIFORNIA INSTITUTE OF TECHNOLOGY

UNIVERSITY OF CALIFORNIA

MONTANA STATE UNIVERSITY

UNIVERSITY OF NEVADA, RENO

NEW MEXICO STATE UNIVERSITY

OREGON STATE UNIVERSITY
UNIVERSITY OF OREGON UNIVERSITY OF SOUTHERN CALIFONIA STANFORD UNIVERSITY UNIVERSITY OF HAWAII UNIVERSITY OF TOKYO UNIVERSITY OF UTAH WASHINGTON STATE UNIVERSITY UNIVERSITY OF WASHINGTON 


\section{Pacific Journal of Mathematics}

\section{Vol. 93, No. $1 \quad$ March, 1981}

Richard Arens, Reducing the order of a Lagrangian $\ldots \ldots \ldots \ldots \ldots \ldots \ldots$

Richard Arens, Manifestly dynamic forms in the Cartan-Hamilton treatment

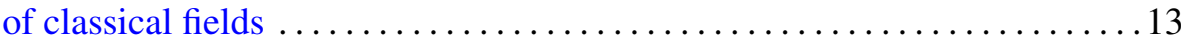

Jimmy T. Arnold, Power series rings over discrete valuation rings $\ldots \ldots \ldots 31$

Charles A. Asmuth and Joe Repka, Supercuspidal components of the

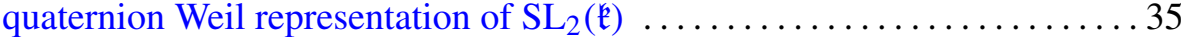

Luis A. Caffarelli and Avner Friedman, Sequential testing of several simple hypotheses for a diffusion process and the corresponding free boundary problem ................................. 49

William B. Jacob, Fans, real valuations, and hereditarily-Pythagorean fields .............................................. 95

W. J. Kim, Asymptotic properties of nonoscillatory solutions of higher order

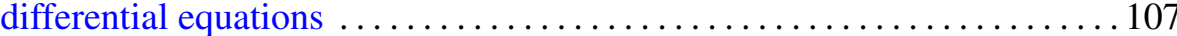

Wayne Steven Lewis, Embeddings of the pseudo-arc in $E^{2} \ldots \ldots \ldots \ldots \ldots 115$

Daniel Alan Marcus, Closed factors of normal Z-semimodules . ......... 121

Mitsuru Nakai and Leo Sario, Harmonic functionals on open Riemann surfaces ............................................. 147

John Currie Quigg, Jr., On the irreducibility of an induced

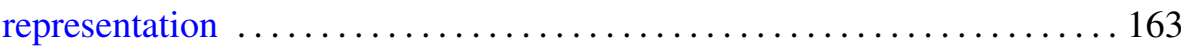

John Henry Reinoehl, Lie algebras and Hopf algebras 181

Joe Repka, Base change for tempered irreducible representations of $\mathrm{GL}(n, \mathbf{R})$

Peter John Rowley, Solubility of finite groups admitting a fixed-point-free automorphism of order $r s t$. I . . . . . . . . . . . . . . . . . . 201

Alan C. Woods, The asymmetric product of three homogeneous linear forms 